D0I: 10.12957/demetra.2015.15116

\title{
Correspondência entre a percepção materna e o estado nutricional de escolares
}

\section{Correspondence between maternal perception and students' nutritional status}

\author{
Luana da Silva Baptista Arpini' \\ André Fiorin Arpini² \\ Geovane Carlos Barbosa ${ }^{3}$ \\ Gláucia Figueiredo Justo ${ }^{4}$ \\ Luciane Bresciani Salaroli ${ }^{5}$ \\ Maria del Carmen Bisi Molina ${ }^{5}$ \\ ' Secretaria de Saúde do Estado do Espírito \\ Santo, Hospital Estadual Infantil Nossa Senhora \\ da Glória. Cariacica-ES, Brasil. \\ 2 Secretaria Municipal de Saúde de Cariacica, \\ Programa de Saúde Mental. Cariacica-ES, Brasil. \\ ${ }^{3}$ Faculdade do Centro Leste, Departamento de \\ Engenharia Biomédica. Serra-ES, Brasil. \\ ${ }^{4}$ Universidade Federal do Rio de Janeiro, \\ Instituto de Estudos em Saúde Coletiva. Rio de \\ Janeiro-RJ, Brasil. \\ ${ }^{5}$ Universidade Federal do Espírito Santo, \\ Departamento de Educação Integrada em Saúde, \\ Curso de Nutrição. Vitória-ES, Brasil. \\ Correspondência / Correspondence \\ Luana da Silva Baptista Arpini \\ E-mail: luanaarpini@hotmail.com
}

\section{Resumo}

Introdução: A percepção materna do estado nutricional do filho (PM), quando discordante do estado nutricional diagnosticado (EN), pode propiciar distúrbios nutricionais. Objetivo: Avaliar a correspondência entre a PM e o EN de escolares, bem como os fatores associados. Métodos: Amostra de 518 escolares de 7 a 10 anos. Foram coletados dados antropométricos para diagnóstico do estado nutricional pelo índice de massa corporal (IMC) e dados socioeconômicos e percepção materna, por meio de entrevista com as mães. Foram aplicados os testes de Kappa ajustado pela prevalência $(k)$ para verificar a concordância entre a PM e o EN, qui-quadrado e exato de Fisher para determinar as diferenças de proporções, e a regressão logística multinomial para ajuste entre as variáveis associadas. Resultado: Foram encontradas maior e menor concordâncias entre PM e EN para magreza e obesidade. Escolares do sexo masculino e aqueles cujas mães se preocupam com o peso têm mais chance de serem percebidos como "abaixo do peso". Conclusão: Foi encontrada concordância substancial entre o estado nutricional e a percepção materna.

Palavras-chave: Percepção. Estado Nutricional. Saúde Escolar. Criança.

\section{Abstract}

Introduction: The maternal perception (MP) of the nutritional status of the child (NS) when mismatched with the diagnosis, can lead to nutritional disorders. Objective: To evaluate the 
correlation between maternal perception and the nutritional status of schoolchildren as well as the associated factors. Methods: A sample of 518 schoolchildren 7-10 years. Anthropometric data were collected for diagnosing nutritional status by body mass index (BMI) and socioeconomic data and maternal perception by means of interviewing the mothers. Prevalence-adjusted Kappa (k) to verify the correspondence between MP and NS, chi-square and Fisher exact tests to determine differences in proportions, and multinomial logistic regression to fit adjust among the associated variables, were applied. Results: Highest and lowest correspondence between the MP and NS were found to thinness and obesity. Male schoolchildren and those whose mothers are concerned with weight are more likely to be perceived as "underweight". Conclusion: Substantial concordance between nutritional status and maternal perception was found.

Key words: Perception. Nutritional Status. School Health. Child.

\section{Introdução}

A percepção é a interpretação que o indivíduo tem do que é observado, por meio de um processo sensorial e $\operatorname{cog}$ nitivo ${ }^{1}$ e, portanto, pode não corresponder à realidade. A percepção materna do corpo do filho, especificamente, quando discordante do estado nutricional diagnosticado, pode acarretar distúrbios nutricionais, ${ }^{2,3}$ influenciar nas práticas de controle alimentar infantil ${ }^{3,4} \mathrm{e}$ dificultar ou inviabilizar o tratamento nutricional. ${ }^{3,5,6}$

De maneira geral, as mães tendem a superestimar o peso das crianças com magreza e subestimar o peso daquelas com sobrepeso e obesidade. ${ }^{3,5,7-11}$ Essa percepção distorcida pode está relacionada a vários fatores. Rietmeijer-Mentink et al. ${ }^{10}$ ao conduzirem metanálise sobre a diferença entre a percepção dos pais e o atual peso da criança, observaram que $63,4 \%$ dos pais de crianças com excesso de peso não reconhecem seus filhos como tal, e esse percentual é elevado para $86 \%$ quando se trata de crianças de 2-6 anos.

Além da idade, também encontramos descritos em outras revisões sistemáticas: sexo, estado nutricional, padrões socioculturais, escolaridade e índice de massa corporal (IMC) maternos, IMC e estado civil paternos e a etnia, como os preditores da percepção materna mais citados na literatura mundial. ${ }^{3,12}$

Apesar da relevância do tema, no Brasil, estudos que propõem avaliar a concordância entre a percepção materna e o estado nutricional de seu filho são escassos, principalmente em região 
rural. Na região urbana do estado do Espírito Santo, observou-se baixa correspondência entre a classificação antropométrica e a percepção materna, especialmente nas crianças obesas, sendo o sexo masculino e a cor da pele da criança não branca os fatores associados. ${ }^{9}$ No entanto, essa associação é ainda desconhecida nos díades mãe-filho na região rural do estado. Assim, o objetivo deste estudo é investigar a correspondência entre a percepção materna e o estado nutricional de escolares de um município rural, bem como os fatores associados.

\section{Métodos}

Estudo transversal, desenvolvido em 2013, a partir da análise dos dados obtidos no projeto "Saúdes - Santa Maria de Jetibá”, realizado em 2009. O município de Santa Maria de Jetibá (SMJ) está localizado na região rural do estado do Espírito Santo, Brasil, e destaca-se como produtor de hortifrutigranjeiros. Foi colonizado por "pomeranos" que, ainda na atualidade, tentam manter a cultura e tradição de suas origens como, por exemplo, a alimentação e o dialeto.

Fizeram parte do estudo escolares de sete a dez anos, de ambos os sexos, matriculados e frequentando as escolas municipais, estaduais ou cooperada, selecionados por amostragem aleatória estratificada, de duplo estágio, levando em consideração o número de alunos matriculados nas escolas por região (perímetros urbano e rural) e o tamanho da escola (pequena: até 50 alunos; média: entre 51 e 200; grande: mais de 200 alunos), sendo proporcional por cotas e esquematizada pelo número de matrículas na escola no ano de 2008. ${ }^{13}$ Foram excluídas crianças cujos dados de peso, altura e percepção materna não estavam completos.

Antes da coleta de dados, estudo piloto foi conduzido com 40 escolares da mesma faixa etária com a finalidade de testar os instrumentos, tempo de coleta, logística e aceitação dos pais para que seus filhos participassem do estudo. Todos os profissionais (acadêmicos dos cursos de Nutrição e Educação Física) que participaram da coleta de dados receberam treinamento teórico e prático para garantir a padronização do protocolo de pesquisa na coleta de dados. Após este estudo, foram realizados os ajustes necessários para iniciar o trabalho de campo no município.

Questionário estruturado foi aplicado aos díades mãe-filho. A percepção materna do estado nutricional do filho foi obtida por meio da pergunta: "Como a senhora acha que seu filho está?", cujas opções de resposta: "abaixo do peso", "peso adequado", "acima do peso" ou "muito acima do peso", correspondiam, respectivamente, aos diagnósticos nutricionais: magreza, eutrofia, sobrepeso e obesidade. A investigação da presença ou ausência de preocupação materna com o peso da criança foi auferida pela pergunta: "A senhora está preocupada ou já se preocupou com o peso de seu filho(a)?”, cujas opções eram sim/não. As variáveis associadas à percepção materna foram: sexo, idade (na data da coleta dos dados), raça/cor, peso ao nascer, localidade da escola, 
estado nutricional materno e infantil, escolaridade e idade maternas, classe socioeconômica e preocupação materna com o peso da criança.

A antropometria das crianças foi realizada na escola e seguiu os procedimentos padronizados pela World Health Organization (WHO), ${ }^{14}$ enquanto os dados socioeconômicos, de saúde, atividade física e alimentação foram obtidos por entrevista com as mães. As crianças foram pesadas descalças e com o mínimo de roupas possível, posicionadas verticalmente no centro da plataforma das balanças digitais da marca Tanita ${ }^{\circledR}$ modelo Family BWF (Tanita, Illinois, EUA), com precisão de 100 gramas, sendo aferido em quilogramas. A estatura foi mensurada em centímetros, com precisão de um milímetro, utilizando estadiômetro portátil da marca Seca ${ }^{\circledR}$ modelo 206 (Seca, Hamburg, BRD). As crianças foram medidas em local plano, com os pés descalços, nádegas e calcanhares contra a parede e olhando para frente. O estado nutricional foi determinado pelo IMC e classificado de acordo com os pontos de corte propostos por Onis et al. em 2007:15 "magreza" $\leq-2$ DP; "eutrofia" $-2<$ DP $\leq+1$; "sobrepeso" > + 1DP; "obesidade" > + 2DP.

O IMC materno foi estimado usando o peso e a altura referidos pela mãe e classificado de acordo com a WHO. ${ }^{16}$ A escolaridade materna correspondeu ao último ano de estudo concluído pela mãe, categorizado em $\leq 3,4-10, \geq 11$ anos. Raça/cor foi classificada por dois entrevistadores independentes e dicotomizada em "branco" e "preto/pardo", localidade da escola por região (rural/ urbana); e a classe socioeconômica foi obtida através do escore de pontuações proposto pela Associação Brasileira de Empresas de Pesquisa $(\mathrm{ABEP})^{17}$ e reclassificada em A+B, C e D+E.

As análises estatísticas foram realizadas utilizando os programas estatísticos IBM $^{\circledR}$ SPSS for Windows versão 20.0 e WINPEPI (PEPI-for-Windows). As diferenças entre proporções foram determinadas pelo teste qui-quadrado e exato de Fisher. Para avaliação da correspondência entre a percepção materna e o estado nutricional, foi utilizado o teste de Kappa ajustado pela prevalência (k), considerando a classificação de Landis \& Koch: ${ }^{18}$ concordância quase perfeita $(0,80-1,00)$, substancial $(0,60-0,79)$, moderada $(0,41-0,59)$, razoável $(0,21-0,40)$ e ruim $(\leq 0,20)$. Para estimar os fatores associados à percepção materna do estado nutricional da criança, foi realizada a análise de regressão logística multinomial, inserindo no modelo as variáveis que apresentaram significância estatística de $20 \%$ na análise univariada. As variáveis que não apresentaram significância estatística foram excluídas do modelo.

Quanto às considerações éticas, foram respeitados os dispositivos da Resolução nº 196/96 do Conselho Nacional de Saúde. O estudo foi conduzido após aprovação pelo Comitê de Ética e Pesquisa (CEP) da Universidade Federal do Espírito Santo (UFES), sob o protocolo nº 60/09 de maio/2009, e após a assinatura do Termo de Consentimento Livre e Esclarecido pelos pais. Os questionários poderiam ser respondidos no idioma pomerano, respeitando e não constrangendo no momento da coleta, visto que os entrevistadores, domiciliados na região, sabiam o dialeto, favorecendo a tradução da entrevista, quando solicitado pelo participante. 


\section{Resultados}

População de estudo composta por 518 escolares, sendo $52 \%$ do sexo masculino, a maioria branco (81,8\%), eutrófico (84\%), classe socioeconômica C (57,4\%) e cujas mães possuem de 4-10 anos de estudo (81,6\%), segundo a tabela 1 .

Tabela 1. Distribuição da amostra e diferença de proporções observadas entre a percepção materna do estado nutricional de escolares e as variáveis independentes $(n=518)$. Santa Maria de Jetibá-ES, 2009.

Percepção materna do estado nutricional do filho

Variáveis

\begin{tabular}{cccc}
\cline { 2 - 3 } Total & $\begin{array}{c}\text { Abaixo do } \\
\text { peso }\end{array}$ & $\begin{array}{c}\text { Peso } \\
\text { adequado }\end{array}$ & $\begin{array}{c}\text { Acima do } \\
\text { valor de } \mathbf{p}^{*}\end{array}$ \\
& peso
\end{tabular}

$\begin{array}{llllllll}\text { n } & \% & \text { n } & \% & \text { n } & \% & \text { n } & \%\end{array}$

\section{Sexo}

Masculino

Feminino

Idade

7 anos

8 anos

9 anos

10 anos

Raça/Cor

Branco

Preto/pardo

Estado Nutricional

Magreza

Eutrofia

Sobrepeso

Obesidade
249
48 ,

49

59,2

40,8
177

178

49,9

50,1

21

22

2148,8

2251,2

$0,191^{*}$

$\begin{array}{llllllll}153 & 29,5 & 41 & 34,2 & 103 & 29,0 & 9 & 20,9\end{array}$

$\begin{array}{llllllll}139 & 26,8 & 30 & 25,0 & 100 & 28,2 & 9 & 20,9\end{array}$

$\begin{array}{llllllll}125 & 24,1 & 27 & 22,5 & 85 & 23,9 & 13 & 30,2\end{array}$

0,500

$101 \quad 19,5 \quad 22$

$67 \quad 18,9 \quad 12$

27,9
374

83

\section{$81,8 \quad 84$}

18,2

84

75,0

261

84,

29

78,4

25,0

47

$15,3 \quad 8$

$0,060^{*}$

21,6 
Percepção materna do estado nutricional do filho

\begin{tabular}{|c|c|c|c|c|c|c|c|}
\hline \multirow[t]{2}{*}{ Variáveis } & \multicolumn{2}{|c|}{ Total } & $\mathbf{A b}$ & do & & ado & \multirow[t]{2}{*}{ valor de $\mathbf{p}^{*}$} \\
\hline & $\mathbf{n}$ & $\%$ & $\mathbf{n}$ & $\%$ & $\mathbf{n}$ & $\%$ & \\
\hline
\end{tabular}

\section{Localidade da}

escola

$\begin{array}{llllllllll}\text { Rural } & 343 & 66,2 & 74 & 61,7 & 246 & 69,3 & 23 & 53,5 & \\ \text { Urbana } & 175 & 33,8 & 46 & 38,3 & 109 & 30,7 & 20 & 46,5 & 0,059^{*}\end{array}$

\section{Peso ao nascer}

Baixo peso

$\begin{array}{llllllll}32 & 6,5 & 8 & 7,1 & 24 & 7,1 & 0 & 0,0\end{array}$

Adequado

$433 \quad 88,4 \quad 102$

91,1

$292 \quad 86,9 \quad 39$

92,9

$0,117^{*}$

Excesso de peso

$\begin{array}{lllll}25 & 5,1 & 2 & 1,8 & 20\end{array}$

$6,0 \quad 3$

7,1

Idade materna

$\leq 30$ anos

12

$>30$ anos

$\begin{array}{ccccccccc}121 & 23,6 & 37 & 31,4 & 76 & 21,6 & 8 & 18,6 & \\ 392 & 76,4 & 81 & 68,6 & 276 & 78,4 & 35 & 81,4 & \end{array}$

\section{Escolaridade}

materna

$\leq 3$ anos

4-10 anos

$\begin{array}{ccccccccr}9 & 1,8 & 3 & 2,5 & 5 & 1,4 & 1 & 2,4 & \\ 416 & 81,6 & 99 & 83,9 & 289 & 82,3 & 28 & 68,3 & 0,127^{*} \\ 85 & 16,7 & 16 & 13,6 & 57 & 16,2 & 12 & 29,3 & \end{array}$

IMC materno

Magreza

Eutrofia

Sobrepeso

$10 \quad 2,8 \quad 5$

$6,0 \quad 5$

$2,1 \quad 0 \quad 0,0$

$18251,1 \quad 48$

$107 \quad 30,1 \quad 1$

Obesidade

$57 \quad 16,0 \quad 14$

$57,1 \quad 12$

$20,2 \quad 75$

2250

$\geq 11$ anos

$\begin{array}{lllll}16,7 & 39 & 16,2 & 4 & 12,9\end{array}$

Preocupação com

o peso do filho

$\begin{array}{llllllllll}\text { Sim } & 271 & 52,8 & 102 & 85,0 & 137 & 38,6 & 32 & 74,4 & <0,001^{* *} \\ \text { Não } & 242 & 47,2 & 18 & 15,0 & 213 & 60,0 & 11 & 25,6 & \end{array}$




\section{Percepção materna do estado nutricional}

do filho

\begin{tabular}{|c|c|c|c|c|c|c|c|c|c|}
\hline \multirow[t]{2}{*}{ Variáveis } & \multicolumn{2}{|c|}{ Total } & \multicolumn{2}{|c|}{$\begin{array}{l}\text { Abaixo do } \\
\text { peso }\end{array}$} & \multicolumn{2}{|c|}{$\begin{array}{c}\text { Peso } \\
\text { adequado }\end{array}$} & \multicolumn{2}{|c|}{$\begin{array}{l}\text { Acima do } \\
\text { peso }\end{array}$} & \multirow[t]{2}{*}{ valor de $\mathbf{p}^{*}$} \\
\hline & $\mathbf{n}$ & $\%$ & $\mathbf{n}$ & $\%$ & $\mathbf{n}$ & $\%$ & $\mathbf{n}$ & $\%$ & \\
\hline \multicolumn{10}{|c|}{$\begin{array}{l}\text { Classe } \\
\text { socioeconômica }\end{array}$} \\
\hline$A+B$ & 20 & 4,3 & 4 & 3,6 & 13 & 4,1 & 3 & 7,9 & \\
\hline $\mathrm{C}$ & 267 & 57,4 & 65 & 58,6 & 181 & 57,3 & 21 & 55,3 & 0,815 \\
\hline $\mathrm{D}+\mathrm{E}$ & 178 & 38,3 & 42 & 37,8 & 122 & 38,6 & 14 & 36,8 & \\
\hline
\end{tabular}

Teste Exato de Fisher *pvalor $\leq 0,10 * * p$ valor $<0,05$

Quanto à percepção materna do estado nutricional infantil, 90,7\% das crianças percebidas como "peso adequado" foram classificadas como eutróficas, 1,1\% como magras e 8,2\% com excesso de peso. Das crianças percebidas como "abaixo do peso", 88,4\% tiveram seu estado nutricional subestimado, sendo que 86,7\% delas eram eutróficas. A maioria das crianças percebidas como "acima do peso" (48,8\%), já estavam obesas, conforme apresentado na tabela 1.

Ao avaliar a correspondência entre a percepção materna e o estado nutricional infantil, foi possível observar maior e menor concordâncias para magreza (77,8\%) e obesidade (3,8\%), segundo a tabela 2.

Tabela 2. Correspondência entre a percepção materna e o estado nutricional de escolares $(\mathrm{n}=518)$. Santa Maria de Jetibá-ES, 2009.

\begin{tabular}{|c|c|c|c|c|c|c|c|c|c|c|}
\hline \multirow{3}{*}{$\begin{array}{l}\text { Estado } \\
\text { nutricional } \\
\text { da criança }\end{array}$} & \multicolumn{8}{|c|}{ Percepção materna do estado nutricional da criança } & & \\
\hline & \multicolumn{2}{|c|}{$\begin{array}{l}\text { Abaixo do } \\
\text { peso }\end{array}$} & \multicolumn{2}{|c|}{$\begin{array}{c}\text { Peso } \\
\text { adequado }\end{array}$} & \multicolumn{2}{|c|}{$\begin{array}{c}\text { Acima do } \\
\text { peso }\end{array}$} & \multicolumn{2}{|c|}{$\begin{array}{c}\text { Muito acima } \\
\text { do peso }\end{array}$} & \multicolumn{2}{|c|}{ Total } \\
\hline & $\mathrm{n}$ & $\%$ & $\mathrm{n}$ & $\%$ & $\mathrm{n}$ & $\%$ & $\mathbf{n}$ & $\%$ & $\mathbf{n}$ & $\%$ \\
\hline Magreza & 14 & 77,8 & 4 & 22,2 & 0 & 0,0 & 0 & 0,0 & 18 & 3,5 \\
\hline Eutrofia & 104 & 23,9 & 322 & 74,0 & 9 & 2,1 & 0 & 0,0 & 435 & 84,0 \\
\hline Sobrepeso & 2 & 5,1 & 24 & 61,5 & 11 & 28,2 & 2 & 5,1 & 39 & 7,5 \\
\hline Obesidade & 0 & 0,0 & 5 & 19,2 & 20 & 76,9 & 1 & 3,8 & 26 & 5,0 \\
\hline Total & 120 & 23,2 & 355 & 68,5 & 40 & 7,7 & 3 & 0,6 & & \\
\hline
\end{tabular}

$\mathrm{k}=0,77$ 
De maneira geral, $67 \%$ das mães perceberam o estado nutricional de seus filhos correspondente ao classificado pelo IMC, enquanto que 30\% subestimaram e 3\% superestimaram (dados não mostrados). Das crianças com excesso de peso, quase a metade das mães (47,7\%) não percebeu que seus filhos estavam acima do peso ou muito acima do peso e 44,6\% das crianças com excesso de peso foram percebidas como "peso adequado".

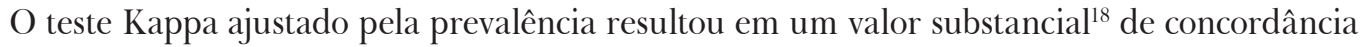
total $(\mathrm{k}=0,77)$, de acordo com a tabela 2. Concordância semelhante foi observada tanto para o sexo feminino $(\mathrm{k}=0,78)$, quanto para o sexo masculino $(\mathrm{k}=0,72)$, ainda que ligeiramente menor para os meninos (tabela 3).

Tabela 3. Correspondência entre a percepção materna e o estado nutricional de escolares, por sexo (n=518). Santa Maria de Jetibá-ES, 2009.

\section{Percepção materna}

\begin{tabular}{|c|c|c|c|c|c|c|c|c|c|c|c|c|c|c|c|c|}
\hline \multirow{3}{*}{$\begin{array}{c}\text { Estado } \\
\text { nutricional } \\
\\
\text { Magreza }\end{array}$} & \multicolumn{8}{|c|}{ Masculino ${ }^{¥}$} & \multicolumn{8}{|c|}{ Feminino $^{\S}$} \\
\hline & \multicolumn{2}{|c|}{$\begin{array}{c}\text { Abaixo do } \\
\text { peso } \\
\text { n (\%) }\end{array}$} & \multicolumn{2}{|c|}{$\begin{array}{c}\text { Peso } \\
\text { adequado } \\
\mathbf{n}(\%)\end{array}$} & \multicolumn{2}{|c|}{$\begin{array}{c}\text { Acima do } \\
\text { peso } \\
\text { n (\%) }\end{array}$} & \multicolumn{2}{|c|}{$\begin{array}{c}\text { Muito } \\
\text { acima do } \\
\text { peso } \\
\text { n (\%) }\end{array}$} & \multicolumn{2}{|c|}{$\begin{array}{c}\text { Abaixo do } \\
\text { peso } \\
\text { n (\%) }\end{array}$} & \multicolumn{2}{|c|}{$\begin{array}{c}\text { Peso } \\
\text { adequado } \\
\text { n (\%) }\end{array}$} & \multicolumn{2}{|c|}{$\begin{array}{c}\text { Acima do } \\
\text { peso } \\
\text { n (\%) }\end{array}$} & \multicolumn{2}{|c|}{$\begin{array}{c}\text { Muito } \\
\text { acima do } \\
\text { peso } \\
\text { n }(\%)\end{array}$} \\
\hline & 7 & 70,0 & 3 & 30,0 & 0 & 0,0 & 0 & 0 & 7 & 87,5 & 1 & 12 & 0 & 0,0 & 0 & 00 \\
\hline Normal & 63 & 27,4 & 163 & 70,9 & 4 & 1,7 & 0 & 0 & 41 & 20,0 & 159 & 77 & 5 & 2,4 & 0 & 0 , \\
\hline Sobrepeso & 1 & 5,9 & 10 & 58,8 & 6 & 35,3 & 0 & 0,0 & 1 & 4,5 & 14 & 63,6 & 5 & 22,7 & 2 & 9,1 \\
\hline Obesidade & 0 & 0,0 & 1 & 8,3 & 11 & 91,7 & 0 & 0,0 & 0 & 0,0 & 4 & 28,6 & 9 & 64,3 & 1 & 7,1 \\
\hline
\end{tabular}

$\$ \mathrm{k}=0,78$

${ }^{\sharp} \mathrm{k}=0,72$.

Quanto à percepção materna em relação aos distúrbios nutricionais de seus filhos, em ambos os sexos, as mães concordaram mais em casos de magreza (70\% para meninos e $87,5 \%$ para meninas). Mães de meninos não perceberam a obesidade em seus filhos, apesar de reconhecerem que seus filhos estavam "acima do peso" (tabela 3).

Observa-se que as crianças do sexo masculino (59,2\%), eutróficas $(86,7 \%)$ e aquelas cujas mães apresentam eutrofia $(57,1 \%)$ foram mais percebidas como "abaixo do peso", enquanto que crianças do sexo feminino $(51,2 \%)$, obesas $(48,8 \%)$ e aquelas cujas mães apresentam sobrepeso $(48,4 \%)$ 
foram mais percebidas como "acima do peso" (tabela 1). Tanto a percepção de baixo-peso quanto a de excesso de peso foram mais frequentes para as crianças brancas $(75,0 \%$ e $78,4 \%)$, escolares de localidade rural (61,7\% e 53,5\%), com peso adequado ao nascer (91,1\% e 92,9\%), em mães com idade superior a 30 anos (68,6\% e 81,4\%), escolaridade materna intermediária (83,9\% e 68,3\%) e naquelas que se preocupam com o peso do filho $(85,0 \%$ e $74,4 \%)$.

A tabela 4 apresenta as variáveis que foram incluídas na análise final $(\mathrm{p}<0,20)$ e que permaneceram associadas significativamente $(\mathrm{p}<0,05)$ à percepção materna após ajuste no modelo de regressão logística.

Tabela 4. Fatores associados à percepção materna do estado nutricional da criança $(n=518)$ : odds ratio ajustado (OR) com respectivo intervalo de confiança (IC95\%). Santa Maria de Jetibá-ES, 2009.

\begin{tabular}{|c|c|c|c|c|}
\hline \multirow{3}{*}{ Variável } & \multicolumn{4}{|c|}{ Percepção materna do peso do filho } \\
\hline & \multicolumn{2}{|c|}{ Abaixo do peso } & \multicolumn{2}{|c|}{ Acima do peso } \\
\hline & OR (IC95\%) & $\begin{array}{c}\text { valor de } \\
\mathbf{p}\end{array}$ & OR (IC95\%) & $\begin{array}{c}\text { valor de } \\
\text { p }\end{array}$ \\
\hline \multicolumn{5}{|l|}{ Sexo } \\
\hline Masculino & $1,653(1,046-2,614)$ & 0,031 & $1,217(0,576-1,217)$ & 0,606 \\
\hline Feminino & 1,000 & & 1,000 & \\
\hline \multicolumn{5}{|l|}{ Preocupação } \\
\hline \multicolumn{5}{|l|}{ Materna } \\
\hline Sim & $9,181(5,278-15,968)$ & $<0,001$ & $2,072(0,893-4,810)$ & 0,090 \\
\hline Não & 1,000 & & 1,000 & \\
\hline
\end{tabular}

*Categoria de referência: percepção materna de peso adequado.

Escolares do sexo masculino (OR=1,653; IC95\%=1,046 - 2,614; $\mathrm{p}=0,031)$ e aqueles cujas mães se preocupam com o peso $(\mathrm{OR}=9,181$; IC95\% $=5,278-15,968 ; \mathrm{p}<0,001)$ têm mais chance de serem percebidos como "abaixo do peso" (tabela 4). 


\section{Discussão}

Os resultados deste estudo corroboram outros que mostram que as mães frequentemente não percebem que seus filhos apresentam um distúrbio nutricional, sobretudo nos casos de excesso de peso. ${ }^{1-3,5,9,19-21}$ Em pesquisa realizada com crianças chilenas, a PM adequada foi altamente associada à prevenção do excesso de peso infantil, diminuindo em 20 vezes a chance da criança ter sobrepeso e obesidade. ${ }^{2}$ Em outro estudo conduzido com crianças alemãs, a probabilidade de a mãe relatar a necessidade de intervenção foi 13 vezes maior para aquelas que identificaram excesso de peso no percentil 75, apesar de a maioria ter indicado que a intervenção deve ser realizada no percentil $>90$ e, $19 \%$ terem dito que até o percentil >97 (obeso severo) não é necessária intervenção. ${ }^{6}$

Os pais de crianças com excesso de peso ou obesos muitas vezes não sabem que seu filho tem um problema de peso ${ }^{8}$ e são mais propensos a reconhecer o excesso de peso quando seus filhos já estão obesos do que quando apresentam sobrepeso. ${ }^{19,22}$ Isso pode refletir uma incapacidade comum de distinguir o peso normal do inadequado, devido ao aumento da prevalência da obesidade. A percepção de peso adequado pode ter se tornado uma percepção mais acima do peso, ou seja, a "normalização" do sobrepeso. ${ }^{19,22-25}$ A ideia de que a criança "gordinha" tem boa saúde e recebe melhor cuidado dos pais pode ser outro preditor dessa distorção da percepção. ${ }^{5}$

Além disso, mães de meninos não perceberam a obesidade em seus filhos, e apresentaram mais chance de percebê-los como "abaixo do peso" do que mães de meninas, como nos resultados encontrados na região urbana do mesmo estado ${ }^{9}$ e nas províncias italianas. ${ }^{19}$ As mães tendem a perceber o baixo-peso em seus filhos e o excesso de peso em suas filhas, e ainda subestimam mais o peso dos meninos. ${ }^{8}$ Isso pode estar relacionado a influências socioculturais e comportamentais, além dos diferentes ideais estéticos. ${ }^{26}$ A percepção materna de corpo ideal para suas filhas está relacionada aos ideais de beleza, os quais correspondem a um corpo magro e bonito, enquanto que, para os meninos, preferem um corpo mais vultoso, que em suas concepções, está relacionado à força e à saúde. ${ }^{27}$

Cabe ressaltar que tanto a magreza quanto o excesso de peso são preocupantes e, portanto, é importante que os pais tenham uma percepção que corresponda ao estado nutricional real de seus filhos, a fim de propiciar intervenções mais precoces, quando necessárias. Todavia, os pais são relutantes em reconhecer potenciais problemas de peso em seus filhos em ambas as extremidades do espectro de peso, e parece que todo o peso é o "peso certo" quando as crianças são saudáveis e felizes. ${ }^{22}$ Por outro lado, pode haver negação ou omissão nos relatos maternos de distúrbio nutricional no filho. ${ }^{27,28}$ As mães sentem-se culpadas quando seu filho tem um agravo nutricional, ${ }^{28}$ uma vez que as pressões sociais e os próprios julgamentos fazem com que elas relacionem essa situação à falta de cuidado materno. ${ }^{27,28}$ 
A distorção da imagem corporal do filho pela mãe é um dos aspectos que podem estar relacionados ao controle da alimentação infantil. ${ }^{3}$ Mães que não percebem o excesso de peso de seus filhos tendem a exercer pressão para que eles comam mais, ${ }^{11}$ o que pode promover a superalimentação da criança e o consequente ganho de peso. ${ }^{3,7}$ Entretanto, os pais que expressam preocupação com o peso do filho são mais propensos a limitar o tempo de tela (televisão, computador, videogame), a incentivar a atividade física e a melhorar a alimentação. ${ }^{29}$ Além disso, mães que se preocupam com o excesso de peso da criança têm mais probabilidade de restringir o consumo de alimentos "não saudáveis". ${ }^{11}$ Estudo de Lindsay et al. ${ }^{27}$ descreve, ainda, que as mães, sobretudo de região rural e indígenas, costumam se preocupar mais com a fome e a desnutrição, devido à associação que fazem com a debilitação da saúde, preferindo ter filhos mais "gordinhos".

Para Campbell et al., ${ }^{30}$ crianças com sobrepeso podem parecer mais bem nutridas e serem percebidas como "mais bem alimentadas", acarretando menor preocupação de seus pais. Esse argumento pode explicar o fato de, no presente estudo, as mães que relataram preocupação com o peso de seus filhos apresentarem nove vezes mais chance de percebê-los como "abaixo do peso" e não "acima do peso"; e de 86,7\% das crianças percebidas como "abaixo do peso" serem eutróficas e, portanto, terem seu estado nutricional subestimado.

A localização da escola (urbana/rural) não permaneceu relacionada à percepção da mãe, ainda que seja possível que os escolares da região mais urbanizada de Santa Maria de Jetibá apresentem melhores condições socioeconômicas e de estilo de vida, quando comparados àqueles de localidade rural. ${ }^{13}$

Também não foi encontrada associação significativa entre a percepção materna do estado nutricional infantil e a escolaridade materna e/ou classe socioeconômica após ajuste. Entretanto, alguns trabalhos apontam o fato de que mães com maior escolaridade e/ou renda parecem ser capazes de identificar o estado nutricional de seus filhos mais próximo do diagnosticado, do que as mães de baixa escolaridade e nível de renda, ${ }^{3,7,9,19}$ alertando para a influência dos determinantes sociais na saúde infantil. Em consonância, estudo conduzido na Holanda, com o objetivo de avaliar a variação étnica na subestimação materna do peso do filho e a influência socioeconômica, verificou que a baixa escolaridade da mãe aumenta quatro vezes mais a chance da criança ter seu peso subestimado. ${ }^{25}$

Outros estudos apontam IMC e idade maternos ${ }^{25}$ e a cor da pele da criança ${ }^{9}$ como preditores da percepção materna, mas tais variáveis não se mantiveram associadas à percepção da mãe, neste estudo. Nesta população rural, a variável raça/cor não é proxy de classe socioeconômica, devido à homogeneidade de indivíduos brancos, diferentemente da população urbana. ${ }^{9}$

O desenho transversal deste estudo nos impede de fazer inferências causais, entretanto, este delineamento possibilita a obtenção de informações precoces para o planejamento de intervenções 
em saúde e para embasar estudos longitudinais. Os resultados se aplicam à população de estudo, em sua maioria composta por indivíduos brancos, eutróficos, classe média/baixa e descendentes pomeranos residentes na região rural do Espírito Santo. Portanto, não são generalizáveis a outros grupos raciais, étnicos, ou geográficos, embora sejam compatíveis com os resultados de outros estudos, inclusive aos observados em investigação similar na região urbana do estado, mas etnicamente diversificada. Isso sugere que, como no estudo de Yao e Hillemeir, ${ }^{21}$ a região de residência do escolar (urbana/rural) não é um preditor da percepção materna do estado nutricional do filho.

Outra limitação é a percepção materna ter sido avaliada por descrição verbal, método considerado subjetivo. Porém, o outro método vastamente utilizado na literatura para avaliação da percepção materna do peso do filho é a escala de silhueta, mas para este ainda não há consenso na literatura de qual instrumento seria o mais adequado. ${ }^{10}$

\section{Conclusão}

Neste estudo, observou-se concordância substancial entre o estado nutricional e a percepção materna, sendo o sexo masculino e a preocupação materna os fatores associados. A percepção materna discordante do estado nutricional pode negligenciar uma intervenção precoce, sobretudo nas crianças com excesso de peso.

Outros estudos são necessários para avaliar os fatores preditores da percepção materna do estado nutricional do filho, bem como a influência dessa problemática nos hábitos de vida e na saúde infantil nos diversos contextos étnicos, sociais e culturais.

\section{Referências}

1. Lara-García B, Flores-Peña Y, Alatorre-Esquivel MA, Sosa-Briones R, Cerda-Flores RM. Evaluation of the maternal perception of childhood overweight-obesity and maternal recognition of health risk in a Mexican border city. Salud Pública de México 2011; 53(3):258-63.

2. Bracho MF, Ramos HE. Percepción materna del estado nutricional de sus hijos: ¿Es un factor de riesgo para presentar malnutrición por exceso? Revista chilena de pediatría. 2007; 78(1):20-7.

3. Arpini LSB, Queiroz DMF, Corrêa MM, Salaroli LB, Molina MCB. Relação entre a percepção materna do peso corporal do filho e as práticas alimentares infantis. Rev. Bras. Pesq. Saúde 2014; 16(3):140-153.

4. Holub SC, Dolan EA. Mothers' beliefs about infant size: associations with attitudes and infant feeding practices. Journal of Applied Developmental Psychology 2012; 33(3):158-64. 
5. Boa-Sorte N, Neri LA, Leite MEQ, Brito SM, Meirelles AR, Luduvice FBS, et al. Maternal perceptions and self-perception of the nutritional status of children and adolescents from private schools. Jornal de Pediatria 2007; 83(4):349-56.

6. Warschburger P, Kröller K. Childhood overweight and obesity: maternal perceptions of the time for engaging in child weight management. BMC Public Health 2012; 12:295.

7. Lopes L, Santos R, Pereira B, Lopes V. Maternal perceptions of children's weight status. Child: Care, Health and Development 2013; 39(5):728-736.

8. Mamun AA, McDermott BM, O’Callaghan MJ, Najman JM, Williams GM. Predictors of maternal misclassifications of their offspring's weight status: a longitudinal study. Int J Obes (Lond). 2008; 32(1):48-54.

9. Molina MCB, Faria CP, Montero P, Cade NV. Correspondence between children's nutritional status and mothers' perceptions: a population-based study. Cad. Saúde Pública. 2009; 25(10):2285-90.

10. Rietmeijer-Mentink M, Paulis WD, van Middelkoop M, Bindels PJE, van der Wouden JC. Difference between parental perception and actual weight status of children: a systematic review. Maternal \& Child Nutrition 2013; 9(1):3-22.

11. Webber L, Hill C, Cooke L, Carnell S, Wardle J. Associations between child weight and maternal feeding styles are mediated by maternal perceptions and concerns. Eur. J. Clin. Nutr. 2010; 64(3):259-65.

12. Chuproski P, Mello DF de. Mother's perception of their children's nutritional status. Rev. Nutrição. 2009; 22(6):929-36.

13. Justo GF, Callo GQ, Carletti L, Molina MCB. Nutritional extremes among school children in a rural Brazilian municipality. Rural Remote Health 2012; 12(4):2220.

14. World Health Organization. Physical status: the use and interpretation of anthropometry indicators of nutritional status [Internet]. Geneva: WHO; 1995. Report No. 854. [acesso em: 3 abr. 2013]. Disponível em: http://whqlibdoc.who.int/trs/WHO_TRS_854.pdf

15. Onis M, Onyango AW, Borghi E, Siyam A, Nishida C, Siekmann J. WHO | Development of a WHO growth reference for school-aged children and adolescents [Internet]. Geneva: WHO; 2007 [Acesso em 3 de abril de 2013]. Disponível em: http://www.who.int/bulletin/volumes/85/9/07-043497/en/

16. World Health Organization. The international classification of adult underweight, overweight and obesity according to BMI. Geneva: WHO; 2004.

17. Associação Brasileira de Empresas de Pesquisa. Critério de classificação econômica Brasil [Internet]. São Paulo: ABEP; 2010. [Acesso em 20 mar. 2013]. Disponível em: http://www.abep.org/criterio-brasil

18. Landis JR, Koch GG. The measurement of observer agreement for categorical data. Biometrics 1977; 33(1):159-74.

19. Binkin N, Spinelli A, Baglio G, Lamberti A. What is common becomes normal: The effect of obesity prevalence on maternal perception. Nutr. Metab. Cardiovasc. Dis. 2013; 23(5):410-416.

20. Hirschler V, Gonzalez C, Talgham S, Jadzinsky M. Do mothers of overweight Argentinean preschool children perceive them as such? Pediatr. Diabetes 2006; 7(4):201-204. 
21. Yao N, Hillemeier MM. Weight status in Chinese children: maternal perceptions and child selfassessments. World Journal of Pediatrics 2012; 8(2):129-35.

22. Petricevic N, Puharic Z, Posavec M, Pavic Simetin I, Pejnovic Franelic I. Family history and parental recognition of overweight in Croatian children. Eur. J. Pediatr. 2012; 171(8):1209-14.

23. Chaparro MP, Langellier BA, Kim LP, Whaley SE. Predictors of accurate maternal perception of their preschool child's weight status among hispanic WIC participants. Obesity 2011;19(10):2026-30.

24. Hager ER, Candelaria M, Latta LW, Hurley KM, Wang Y, Caulfield LE, et al. Maternal perceptions of toddler body size: accuracy and satisfaction differ by toddler weight status. Arch. Pediatr. Adolesc. Med. 2012; 166(5):417-22.

25. Hoog MLA, Stronks K, van Eijsden M, Gemke RJBJ, Vrijkotte TGM. Ethnic differences in maternal underestimation of offspring's weight: the ABCD study. Int. J. Obes. (Lond). 2012; 36(1):53-60.

26. Gualdi-Russo E, Manzon VS, Masotti S, Toselli S, Albertini A, Celenza F, et al. Weight status and perception of body image in children: the effect of maternal immigrant status. Nutr. J. 2012; 11:85.

27. Lindsay AC, Machado MT, Sussner KM, Hardwick CK, Franco Sansigolo Kerr LR, Peterson KE. Brazilian mothers' beliefs, attitudes and practices related to child weight status and early feeding within the context of nutrition transition. J. Biosoc. Sci. 2009; 41(1):21-37.

28. Silva DA, Andrade GN, Ferreira FMR, Andrade E, Madeira AMF. Percepcion materna sobre el disturbio nutricional del hijo: un estudio comprensivo; Maternal perceptions about the nutritional disorder of the child: a comprehensive study. REME Rev. Min. Enferm. 2011; 15(4):498-503.

29. Moore LC, Harris CV, Bradlyn AS. Exploring the relationship between parental concern and the management of childhood obesity. Matern Child Health J. 2012; 16(4):902-8.

30. Campbell MW-C, Williams J, Hampton A, Wake M. Maternal concern and perceptions of overweight in Australian preschool-aged children. Med. J. Aust. 2006; 184(6):274-7.

Recebido: $14 / 2 / 2015$

Revisado: $25 / 5 / 2015$

Aprovado: 12/10/2015 